\title{
Mapping urban greenery to create the optimal cooling effect model against solar radiation under the conditions of a smart city
}

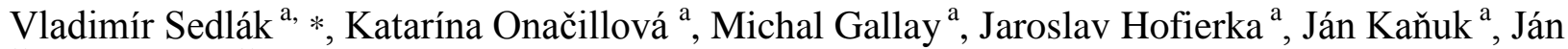 \\ Šašak ${ }^{a}$, Jozef Šupinskýa ${ }^{\mathrm{a}}$ \\ ${ }^{a}$ Pavol Jozef Šafárik University in Košice,vladimir.sedlak@upjs.sk, onacillova.katarina@gmail.com,michal.gallay@upjs.sk, \\ jaroslav.hofierka@upjs.sk,jan.kanuk@upjs.sk,jan.sasak@upjs.sk,jozef.supinsky@upjs.sk \\ * Corresponding author
}

Keywords: Mapping, Urban greenery, LiDAR, Cooling effect model, Smart city

\begin{abstract}
:
Current climate changes on a global scale require an optimal estimate of heat transfer in a complex urban environment as a part of the requirements for optimal urban planning in the conditions of a smart city. Urban greenery has a considerable impact on the cooling of the urban environment during thermal waves. Sentinel-2 as an Earth observation mission developed by the European Space Agency as part of the Copernicus Programme to perform terrestrial observations in support of various services could become a potential means also for quantified assessment of different urban scenarios where vegetation plays an essential role. The Sentinel-2 data provide higher spatial and temporal resolution than other similar missions allow.
\end{abstract}

The presented research study is aimed at exploiting the potential of Sentinel-2 in simulating the cooling effect of urban greenery as part of smart city mapping in assessing the quality of life of its inhabitants. The main objective of the research study is to define a methodical approach for spatial surface temperature modelling in selected urban areas based on the solar radiation modelling and parameterization of the land cover properties from the Sentinel-2 data. While solar irradiation can be accurately calculated at a fine scale using virtual 3D city models, it is difficult to find other important parameters for ground surface modelling such as surface thermal emissivity, broadband albedo and evapotranspiration. The research study was tested and verified in 4 sq. km urban area in the selected central parts of the city of Košice in Slovakia (Figure 1). For a detailed survey, four sites (site 1 - Moyzesova Street, site 2 - Historical centre, site 3- City park, site 4 - Hvozdíkov park) were chosen in the central city area. The virtual 3D urban model was created from the airborne LiDAR (Light Detection And Ranging, hereinafter referred to as the lidar) and photogrammetric data obtained in a single mission.

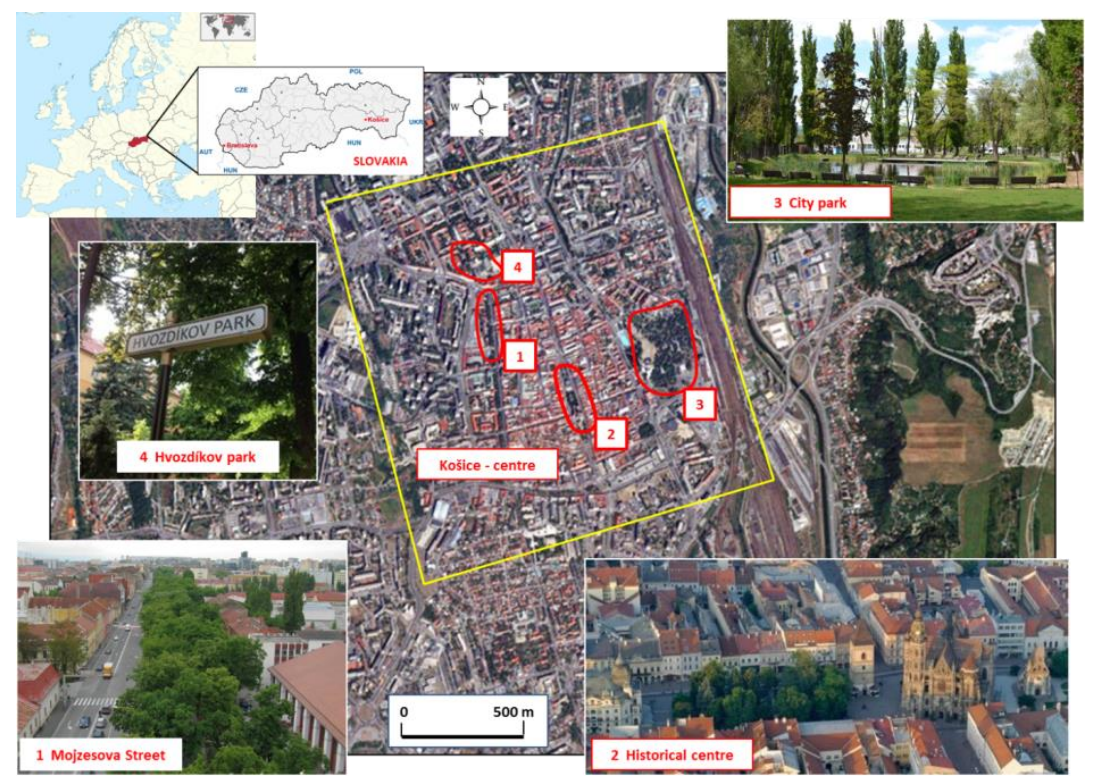

Figure 1. Location of the study area in the city of Košice, Slovakia with the surveyed sites. The yellow line outlines the area subject to airborne lidar and photogrammetric mapping in a single mission, time series of the Sentinel-2 image coverage. The red outline delineates four sites selected for repeated terrestrial laser scanning of urban vegetation. The background maps are (C) Copernicus, Sentinel-2A image acquired on September 7, 2016. 
The aim of the research study was to assess the feasibility of using virtual 3D city models and multispectral satellite images to approximate surface temperature dynamics by modelling of the spatial distribution of solar radiation and land surface characteristics in a complex urban environment. A time-series of the Sentinel-2 data was collected for comparison with the reference time series of the terrestrial lidar (TLS - Terrestrial Laser Scanning) data on urban greenery on four selected urban areas of the city of Košice. Between the vegetation metrics, the statistical linear relationship derived from the Sentinel-2 and TLS data was defined. Based on terrain mapping, a geobotanic database of urban trees was created. The algorithmic structure of a toolbox for the land surface temperature modelling in the opensource GRASS GIS was developed based on the Stefan-Boltzmann law and Kirchhoff rule.

This research study has highlighted how the Sentinel-2 data can be used to estimate of the broad-band albedo, surface emission, and solar transmittance to the vegetation of urban greenery. The main benefit of the research study is the developed algorithm for estimation of the land surface temperature in a GIS environment that provides a unique platform for integrating different types of data-sets to become usable in urban planning and for exploitation of the Sentinel-2 data in mitigation of a negative impact of the urban extreme heat islands on the quality of life of inhabitants. The resulting LST (Land Surface Temperature) was calculated for four scenarios using the detail of the study area of the site 1 (Figure 2) and whole study are (Figure 3) demonstrate. These figures also show the cooling effect of urban trees and shrubs.
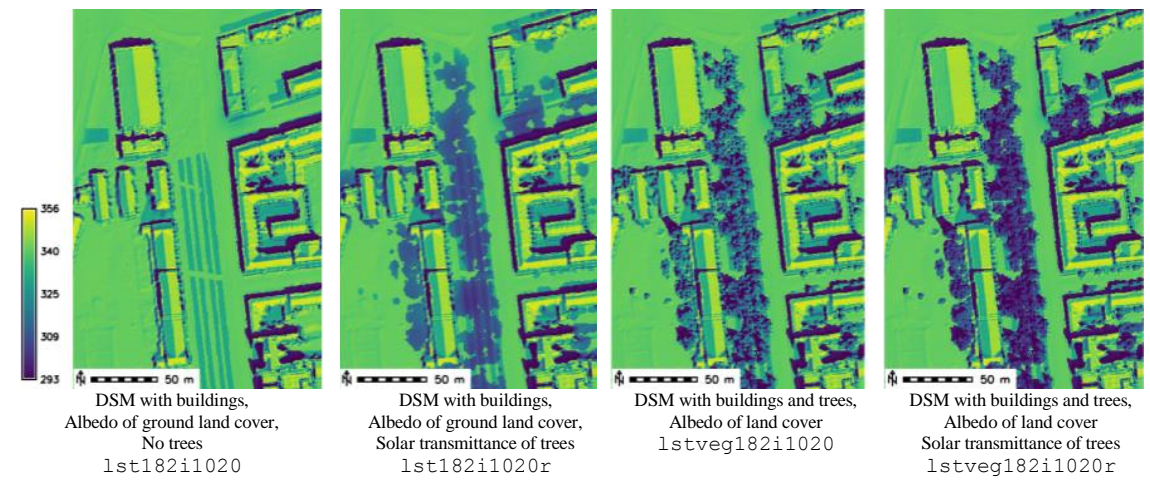

Figure 2. Modelled temperature of ground surface (kelvins) in site 1 - Moyzesova Street, without and with implementing vegetation transmittance downscaled from linear regression model of the Sentinel-2_NDVI (Normalized Difference Vegetation Index) vs. LAI (Leaf Area Index)_TLS for June 30, 2016 at 9:20 a.m. UTC (United Time Coordinated)/10:20 a.m. local time. In the first two images DSM (Digital Surface Model) of terrain and building surface is used and albedo is derived for the ground land cover below the trees. In the latter two images, DSM of terrain, buildings and trees is used and albedo of the land cover canopy surface was used.
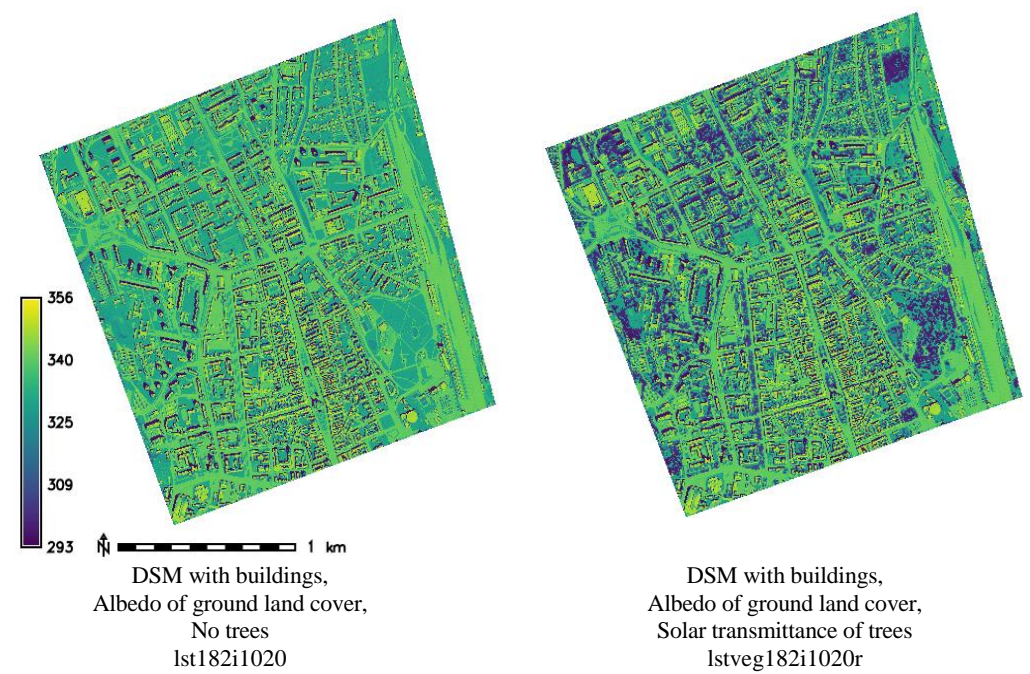

$$
\begin{gathered}
\text { DSM with buildings, } \\
\text { Albedo of ground land cover, } \\
\text { Solar transmittance of trees } \\
\text { lstveg } 182 \mathrm{i} 1020 \mathrm{r}
\end{gathered}
$$

Figure 3. Modelled temperature of ground surface (kelvins) in the whole entire study area of the city of Košice without and with implementing vegetation transmittance downscaled from the linear regression model of the Sentinel-2_NDVI vs. LAI_TLS for June 30, 2016 at 9:20 a.m. UTC/10:20 a.m. local time.

The presented research study was granted by the projects APVV SK-CN-RD-18-0015, KEGA 007UPJŠ-4/2017 and VEGA 1/0839/18 solved at the Institute of Geography of the Faculty of Science, Pavol Jozef Šafárik University in Košice, Slovakia. 